\title{
AS CARACTERÍSTICAS DOS DISCENTES DE CIÊNCIAS CONTÁBEIS E AS ESTRATÉGIAS METACOGNITIVAS DE APRENDIZAGEM AUTORREGULADA
}

http://dx.doi.org/10.5902/2318133842375

\author{
Thiago Bruno de Jesus Silva ${ }^{1}$ \\ Marcelo Machado de Freitas ${ }^{2}$ \\ Jonatas Dutra Sallaberry ${ }^{3}$ \\ Leonardo Flach ${ }^{4}$
}

\begin{abstract}
Resumo
Neste texto apresenta-se resultados de pesquisa que teve como objetivo analisar a relação entre as estratégias de aprendizagem autorregulada e as características individuais de discentes do curso de Ciências Contábeis. Aplicou-se modelagem de equações estruturais com mínimos quadrados parciais - PLS/SEM - em amostra de 364 estudantes por meio do instrumento de Zimmermann e Ponz (1986). Os resultados revelaram as estratégias empregadas, bem como a relação entre as características individuais às estratégias, reforçam a premissa de que estudantes com diferentes características empregam diferentes estratégias. A discussão dos resultados apresenta evidências que podem contribuir com o processo pedagógico de ensino, bem como fornece insights para a explicar problemas da literatura sobre a temática de aprendizagem autorregulada.
\end{abstract}

Palavras-chave: ensino; aprendizado; memorização; desempenho.

\section{THE CHARACTERISTICS OF ACCOUNTING STUDENTS AND METACOGNITIVE STRATEGIES FOR SELF-REGULATED LEARNING}

\begin{abstract}
The research aimed to analyze the relationship between self-regulated learning (SRL) strategies and the individual characteristics of students in the Accounting course. Structural equation modeling with partial least squares - PLS/SEM - was applied to a sample of 364 students using the instrument by Zimmermann and Ponz (1986). The results revealed the strategies employed, as well as the relationship between the individual characteristics to the strategies, reinforce the premise that students with different characteristics employ different strategies. The discussion of the results presents evidence that can contribute to the pedagogical process of teaching as well as provides insights to explain problems in the literature on the theme of self-regulated learning. Key-words: teaching; learning; memorization; performance.
\end{abstract}

\footnotetext{
1 Universidade Federal da Grande Dourados, Brasil. E-mail: thiagobsilva@ufgd.edu.br.

2 Universidade Federal de Santa Catarina, Brasil. E-mail: mmf.marcelofreitas@gmail.com.

3 Universidade Federal de Santa Catarina, Brasil. E-mail: jonatas.sallaberry@hotmail.com.

${ }^{4}$ Universidade Federal de Santa Catarina, Brasil. E-mail: leonardo.flach@gmail.com.

\begin{tabular}{|l|l|l|l|l} 
Regae: Rev. Gest. Aval. Educ. & Santa Maria & v. 9 & n. 18 & Pub. contínua 2020
\end{tabular} p. $1-17$
} 


\section{Introdução}

o reconhecer a evolução na profissão contábil o Instituto Americano de
Contadores Públicos Certificados (AICPA, 2010) e a Associação Americana
de Contabilidade (AAA, 2012) destacam a importância de se entender a aprendizagem como um processo vitalício que requer autoconsciência e se estende além do âmbito acadêmico. Neste sentido, o desenvolvimento de habilidades metacognitivas de aprendizagem autorregulada possibilita melhor adaptação aos contextos de mudanças acadêmicas e profissionais em contabilidade (Smith, 2001; Beck, 2013).

A definição comum descreve a aprendizagem autorregulada como o processo de ser metacognitiva, motivacional e comportamental ativo no seu próprio processo de aprendizagem (Zimmerman, 1986; 2001). A aprendizagem autorregulada envolve uma abordagem de autoconsciente e intencional para a realização acadêmica ou o sucesso da aprendizagem. Os alunos bem-sucedidos são aqueles que possuem alto grau de autorregulação metacognitiva, autorregulação motivacional e autorregulação comportamental (Schleifer; Dull, 2009).

O foco desta investigação foi 0 aspecto metacognitivo de aprendizagem autorregulada e suas interrelações com características censitárias dos acadêmicos. Deste modo, a metacognição é descrita como o conhecimento que o sujeito tem sobre seu próprio conhecimento ou processos mentais (Flavell, 1976). Contudo, outros estudos desenvolvidos definiram metacognição como o comando dos próprios processos e produtos cognitivos. No entanto, com uma análise mais detalhada destas definições para o termo metacognição, é possível encontrar uma essência comum no que diz respeito ao controle cognitivo preenchido por um conjunto de mecanismos internos que promovam, produzam, gravam e consideram informação, bem como monitoram e autorregulam seu próprio processamento intelectual (Lima Filho, Lima; Bruni, 2015).

Embora haja estudos que investigaram o papel da aprendizagem autorregulada, como Schleifer e Dull (2009), Byrne e Flood (2009), Becker (2013), Lima Filho, Lima e Bruni (2015), Silva (2016) e Silva, Lay, Biavattti e Haag (2016), Kraus, Silva e Zonatto (2017) e Silva e Biavatti (2018) estes não consideraram o impacto que o nível de uso das estratégias metacognitivas de aprendizagem autorregulada pode ocasionar no desempenho acadêmico, o que foi sugerido por Scheleifer e Dull (2009), e também não levam em conta as diferentes características censitárias dos alunos. Isso porque, conforme apontam diferentes pesquisas, algumas características são fundamentais para se compreender adequadamente 0 potencial de aprendizagem e 0 desempenho acadêmico que os estudantes podem ter (Coleman et al., 1968, Giménez, Prior \& Theme, 2007).

Neste contexto, o problema de pesquisa revela-se pelo fato de que estratégias de aprendizagem aplicáveis a um aluno pode não ser adequada a outros alunos, demandando um atendimento especial ao discente, de acordo com a sua dificuldade (Sallaberry; Sallaberry, 2012). Diante disso, qual a relação entre as características individuais dos alunos as estratégias metacognitivas empregadas para o aprendizado?

É necessário entender como os alunos podem aplicar melhor as estratégias de aprendizagem auto-reguladas para obter sucesso acadêmico (Broadbent; Poon, 2015). Silva, Sallaberry, Freitas e Flach (2019) indicam que o desempenho está associado apenas a algumas estratégias de aprendizagem, mas não é conhecido na literatura a 
validação empírica das características do indivíduo que levam a adoção de determinada estratégia. Assim, essa contribuição torna-se relevante à luz do reconhecimento de que os estudantes, futuros profissionais em contabilidade, necessitam desenvolver habilidade de pensamento crítico, de aprendizagem continuada e a capacidade de fornecer aconselhamento de qualidade para a tomada de decisões. A esse respeito tem-se observado um número ainda incipiente de estudos sobre a temática (Ravenscroft; Waymire; West, 2012; Lima Filho; Lima; Bruni, 2015; Silva, 2016). Como já se tem demonstrado na literatura, estratégias metacognitivas de aprendizagem autorreguladas afetam, de forma positiva, o desempenho acadêmico e por esse motivo, torna-se importante estudar quais características pessoais interferem no uso dessas estratégias.

\section{Fundamentação teórica}

Os graduados em contabilidade entram numa profissão caracterizada pelo aumento da complexidade associada com um corpo crescente de conhecimento (Ravenscroft, Waymire; West, 2012). Os professores enfrentam a cada dia mais desafios para transmitir o conhecimento aos discentes (Farias et al., 2019). Organismos internacionais defendem a aprendizagem contínua e acrescentam que o sucesso na profissão depende da capacidade dos profissionais e acadêmicos em avaliar e regular a sua própria aprendizagem neste âmbito de constante mudança (Bolzan et al., 2020; AICPA, 2010; AAA, 2012).

Para Zimmerman (1990) os aprendizes autorregulados são definidos como participantes metacognitivos, motivacionais e com comportamento ativo em seus próprios caminhos do conhecimento. A aprendizagem autorregulada se refere ao processo de refletir sobre como se pensa, aprende, raciocina e resolve problemas (National Research Council, 2001). Os estudantes e profissionais da área de contabilidade devem desenvolver visão precisa de suas habilidades técnicas e interpessoais, avaliar continuadamente suas próprias habilidades, buscar oportunidades de melhoria e se comprometer com a aprendizagem ao longo da vida (Ravenscroft; Waymire; West, 2012).

Zimmerman (2000) considera importante o aspecto social na evolução da aprendizagem. Em seu trabalho $o$ autor analisa os processos psicológicos implicados nas diversas fases da autorregulação: fase de antecipação e preparação: planejamento; fase de execução e controle: realização; fase de autorreflexão: avaliação. O seu instrumento tem sido bastante utilizado em pesquisas com estudantes sobre a estratégias de aprendizagem e a motivação em diversos países (Chen, 2002; Beck, 2013).

Zimmerman e Ponz (1986) desenvolveram framework com 14 possíveis estratégias do aprendizado autorregulado. Para estes autores o uso destas estratégias confere ao aluno um valioso ferramental, pois sua utilização está correlacionada com os índices de sucesso acadêmico e com a opinião dos docentes acerca do seu grau de autorregulação em sala de aula. As estratégias são: a) autoavaliação; b) organização e transformação; c) estabelecimento de objetivos e planejamento; d) procura de informação; e) apontamentos; f) estrutura ambiental; g) autoconsequências; h) repetição e memorização; i) ajuda de professores; j) ajuda de pares próximos; k) ajuda de especialistas; I) revisão das anotações; m) revisão de testes e n) revisão da bibliografia. 
O aluno com grau desejável para autorregular sua aprendizagem tende a utilizar mais categorias de estratégias autorregulatórias: auto avaliação, organização e transformação, planejamento e elaboração de metas, busca de informações, monitoramento e manutenção das anotações, estruturação do ambiente, auto consequência, ensaio e memorização, pedido de ajuda aos pares, aos professores, à família, revisão de provas, de anotações e de textos (Zimmermann, 1986) e gerenciamento do tempo (Rosário, 2004). Além disso, alunos que tendem a apresentar sua aprendizagem autorregulada exibem maior rendimento acadêmico e melhores percepções de auto eficácia (Zimmermann; Martinez-Pons, 1990).

Tuysuzoglu (2011) argumenta que o perfil do aluno autorregulado é de uma pessoa ativa e construtiva que, portanto, estabelece metas para o seu aprendizado, acompanhando tal processo e que busca regular e controlar a sua cognição, motivação e comportamento, guiado e limitado por seus objetivos e características contextuais do ambiente. Os resultados dos estudos demonstram que existe diferença entre os alunos que utilizam as estratégias metacognitivas de aprendizagem autorregulada. Segundo Zimmermann (2001) os autorregulados são decididos, estratégicos, persistentes e capazes de avaliar os seus processos, já os não autorregulados não possuem objetivos educacionais definidos, possuindo desta forma, uma maior dependência cognitiva. Destaca-se que o número de utilização de estratégias de aprendizagem é maior para alunos concluintes e que a prática de ensino, como o método educacional, pode promover o comportamento.

A consciência e o controle dos pensamentos mentais, a metacognição, sinaliza ao aluno os pontos fracos aos quais ele deve dispender mais esforço para entender o assunto. Carson (2011), Chang (2007) e Puzziferro (2008) corroboraram essa perspectiva de melhora o desempenho acadêmico, enquanto Chang (2010), Cho e Shen (2013), Hodges e Kim (2010) e Klingsieck et al. (2012) não encontraram significância nessa perspectiva.

O tempo é fator crucial para o bom desempenho acadêmico, para o aprendizado, enquanto a gestão do tempo refere-se à capacidade de planejar o tempo e as tarefas do estudo (Broadbent; Poon, 2015; Effeney, Carroll; Bahr, 2013). Carson (2011) e Puzziferro (2008) encontraram uma relação positiva entre a gestão do tempo e o desempenho com fraca relação, enquanto na amostra das pesquisas de Klingsieck et al. (2012) não foi possível identificar significância estatística.

O engajamento na busca dos objetivos ou esforço refere-se à capacidade de persistir quando confrontada com desafios acadêmicos (Richardson et al., 2012) é dos fatores que pode melhorar o desempenho do discente. Essa perspectiva foi confirmada por Carson (2011), Cho e Shen (2013) e Puzziferro (2008), enquanto ChanLin (2012) não confirmaram essa relação na sua amostragem.

A colaboração entre os alunos ajuda na aprendizagem (Effeney et al., 2013), o que foi confirmado por ChanLin (2012) e Puzziferro (2008). Já a obtenção de assistência de instrutores com o objetivo de superar desafios acadêmicos (Richardson et al., 2012) encontrou uma fraca associação com o desempenho (Puzziferro, 2008). 
A tomada de apontamentos ou sintetização de conteúdo em resumos refere-se à capacidade de fundir informações novas e existentes com o objetivo de lembrar o novo material (Richardson et al., 2012) foi identificado com relação positiva fraca (Puzziferro, 2008), enquanto outros dois estudos não encontraram uma relação significativa (Klingsieck et al., 2012; Wang; Wu, 2008). Pode-se destacar também a capacidade de destacar pontos principais durante o aprendizado (Effeney et al., 2013) por meio de gráficos e tabelas, com relação positiva fraca para Puzziferro (2008), e não significativa para Klingsieck et al. (2012).

A memorização ou aprendizado por repetição (Effeney et al., 2013), um aluno que assiste a um vídeo explicativo várias vezes, também revela-se com fraca associação no estudo de Puzziferro (2008), enquanto não foi corroborado em Klingsieck et al. (2012) e Wang e Wu (2008). Interessante destacar que o pensamento crítico ou capacidade de examinar cuidadosamente o material de aprendizagem (Richardson et al., 2012) também foi identificado com influência positiva e fraca (Puzziferro, 2008) em relação do desempenho acadêmico.

A conjugação dessas estratégias de aprendizado auto-reguladas combinadas demonstraram-se associadas às estratégias de aprendizado autoregulado (Broadbent; Poon, 2015) por meio de metaanálise.

Para verificação empírica utilizou-se do instrumento elaborado por Zimmermann e Ponz (1986) que, reduzido por Lima Filho et al. (2015), inclui dez afirmações relativas ao uso das estratégias metacognitivas de aprendizagem autorregulada, conforme descrito no quadro 1.

\section{Quadro 1 -}

Afirmações e estratégias metacognitivas de aprendizagem autorregulada.

\begin{tabular}{|c|c|}
\hline Estratégia & Função \\
\hline $\begin{array}{l}\text { 1. Avalio o meu desempenho, vejo o que devo melhorar e procuro } \\
\text { superar dificuldades detectadas }\end{array}$ & Avaliação \\
\hline $\begin{array}{l}\text { 2. Procuro sempre elaborar um plano (esquema) antes de iniciar um } \\
\text { trabalho }\end{array}$ & \multirow{3}{*}{ Planejamento } \\
\hline $\begin{array}{l}\text { 3. Se tenho prova, começo a estudar o mais cedo possível, para } \\
\text { ficar descansado e tranquilo no dia. }\end{array}$ & \\
\hline $\begin{array}{l}\text { 4. Antes de iniciar um trabalho, recorro sempre à biblioteca (e outros } \\
\text { meios de pesquisa seja físico ou digital) parar separar o máximo de } \\
\text { informação sobre o tema. }\end{array}$ & \\
\hline $\begin{array}{l}\text { 5. Sempre procuro anotar o máximo de apontamento de um texto } \\
\text { lido ou da aula expositiva do professor. }\end{array}$ & \multirow{4}{*}{ Execução e controle } \\
\hline $\begin{array}{l}\text { 6. Para ter melhor concentração, procuro sempre ambiente que não } \\
\text { proporcione distração. }\end{array}$ & \\
\hline $\begin{array}{l}\text { 7. Quando faço uma prova, se ocorrer bem, ofereço-me } \\
\text { recompensa; caso ocorra o contrário, abro mão de algo que tanto } \\
\text { queria. }\end{array}$ & \\
\hline $\begin{array}{l}\text { 8. Utilizo estratégias para memorizar o assunto (ou fórmulas), até } \\
\text { saber de cor, do assunto a ser estudado. }\end{array}$ & \\
\hline $\begin{array}{l}\text { 9. Quando surge dificuldade e não consigo resolver sozinho, busco } \\
\text { ajuda externa (professor, colegas, outros). }\end{array}$ & \\
\hline
\end{tabular}


10. Após conclusão de um trabalho acadêmico, eu o reviso para ter a certeza de que esteja correto.

Fonte: Zimmermann e Ponz (1986) adaptado por Lima Filho et al. (2015)

Destaca-se que no campo das estratégias metacognitivas de aprendizagem autorregulada poucos estudos investigaram o tema na educação contábil, como são os casos de Sperling et al., (2004), Schleifer e Dull (2009), Byrne e Flood (2009), Becker (2013), Ravenscroft, Waymire e West, (2012), Lima Filho et al. (2015), Silva et al., (2016), Kraus, Silva e Zonatto (2017) e Silva e Biavatti (2018). A convergência entre tais pesquisas está no fato de que a predominância que investiga o nível de utilização das estratégias metacognitivas de aprendizagem autorregulada pelos estudantes. Com exceção de Schleifer e Dull (2009), que discutiram a associação entre a metacognição dicotomizada em conhecimento metacognitivo e metarregulação com o desempenho acadêmico, e Ravenscroft, Waymire e West, (2012) que pesquisaram a metacognição associada ao desempenho, erro de calibração e mentalidade.

Contudo, os resultados sobre 0 nível de estratégias metacognitivas de aprendizagem autorregulada e o desempenho foram inconclusivos ao considerar que Schleifer e Dull (2009) encontraram que maior conhecimento metacognitivo estava associado a notas mais altas, enquanto para cada nível de metaconhecimento a metaregulação dos alunos estava negativamente relacionada às notas dos cursos. Já Ravenscroft, Waymire e West, (2012) encontraram que alunos que superestimam suas habilidades metacognitivas tiveram baixo desempenho acadêmico.

Enfim, não há consenso na literatura sobre a relação entre o desempenho e o nível de estratégias metacognitivas utilizadas pelos alunos. Entretanto, existe a crença entre os professores que habilidades metacognitivas estão relacionadas ao desempenho acadêmico (Ravenscroft; Waymire; West, 2012; Sperling et al., 2004).

Outro ponto que pode ser levado em consideração e que pode impactar os resultados de um estudo autorregulado são as características e condições a que os estudantes estão sujeitos. Uma extensa e reconhecida pesquisa que abordou o tema foi o Relatório de Coleman (Coleman et al., 1966), que dentre os diversos achados identificou que características pessoais e sociais são importantes variáveis explicativas para os resultados acadêmicos. Exemplos dessas características são os recursos tangíveis e intangíveis que os acadêmicos possuem, como por exemplo boas condições de estudo em casa, disponibilidade de tempo para estudar, possuir pais com maior nível educacional, possuir livros em casa etc.

Silva et al. (2009) indicam que o desempenho está associado apenas a algumas estratégias de aprendizagem, mas não é conhecido na literatura a validação empírica das variáveis que levam a adoção de determinada estratégia. Portanto, este contexto incentivou esta investigação, no qual se busca oferecer explicações validadas sobre as variáveis que induzem a escolha de determinadas estratégias de aprendizagem entre os estudantes de contabilidade, levando em consideração diferentes características censitárias dos alunos. 


\section{Trajetória metodológica}

Para analisar relação entre o desempenho acadêmico com o nível do uso das estratégias metacognitivas de aprendizagem autorregulada pelos estudantes de contabilidade, considerando os efeitos das características censitárias dos alunos, adotouse procedimentos documentais e utilização de dados provenientes de questionário aplicados aos discentes. A abordagem do estudo foi quantitativa, sendo utilizados procedimentos estatísticos para responder a questão de pesquisa.

A pesquisa foi operacionalizada com aplicação de survey com o instrumento de Zimmermann e Ponz (1986) adaptado por Lima Filho et al. (2015) que inclui dez afirmações relativas ao uso das estratégias metacognitivas. Os discentes foram convidados a basear suas respostas em sua experiência e deveriam atribuir uma nota em resposta ao quesito de frequência do emprego das referidas estratégias em escalas tipo Likert de sete pontos. Após obter a nota de utilização das estratégias metacognitivas de aprendizagem autorregulada, buscou-se analisar a relação entre 0 desempenho acadêmico e o nível de uso das estratégias.

Para se compreender o efeito das características censitárias dos respondentes sobre as estratégias de ensino autorregulado, múltiplas regressões foram realizadas tendo como variáveis dependentes cada uma das dez estratégias. As variáveis independentes analisadas foram o desempenho dos acadêmicos, possuir ou não ou bolsa de estudos, idade, gênero, tempo de estudo diário e por último um conjunto de itens que avaliou a autopercepção relacionada a síndrome do impostor.

A amostra do estudo compreendeu 364 estudantes do curso de Ciências Contábeis na modalidade de ensino presencial de três instituições de âmbito federal que responderam totalmente 0 instrumento de pesquisa. A coleta foi realizada entre 22 de janeiro de 2018 a 30 de novembro de 2018. Os questionários foram entregues em formato impresso e mediante formulário eletrônico enviado por e-mail, resultando na amostra de estudantes conforme características da tabela 1.

Tabela 1 -

Análise descritiva dos dados.

\begin{tabular}{c|c|c|c|c}
\hline \multicolumn{2}{c|}{ Amostra } & \multicolumn{3}{c}{$\begin{array}{c}\text { Gênero } \\
\text { Feminino }\end{array}$} \\
\hline $\mathrm{Fi}$ & Total & & Masculino \\
\hline $\mathrm{Fi} \%$ & 364 & $\mathrm{Fi}$ & 223 & 141 \\
\hline \multicolumn{2}{c|}{ Idade } & $\mathrm{Fi} \%$ & 61,26 & 38,74 \\
\hline
\end{tabular}

\begin{tabular}{c|c|c|c|c} 
Até 20 & De 21 a 25 & De 26 a 30 & De 31 a 35 & Acima de 35 \\
\hline 114 & 136 & 56 & 32 & 25 \\
\hline 31,40 & 37,47 & 15,43 & 8,82 & 6,89 \\
\hline \multicolumn{4}{|c}{ Semestre }
\end{tabular}

\begin{tabular}{c|c|c|c|c|c} 
& 20 & $4^{\circ}$ & 60 & 80 & 3ㅇ \\
\hline $\mathrm{Fi}$ & 137 & 77 & 66 & 40 & 22 \\
\hline $\mathrm{Fi} \%$ & 37,95 & 21,33 & 18,28 & 11,08 & 6,09 \\
\hline
\end{tabular}




\begin{tabular}{c|c|c|c|c}
\hline \multicolumn{4}{c}{ Mesempenho acadêmico } \\
Média & \multicolumn{2}{c|}{ Mánimo } & Desvio Padrão \\
\hline 6,59 & \multicolumn{2}{|c|}{1,0} & 9,5 & 1,71 \\
\hline \multirow{3}{*}{ não estudo } & menos de 30 & aproximadamente & entre 2 ou 3 horas & mais de 4 horas ao \\
& minutos ao dia & 1 hora ao dia & ao dia & dia \\
\hline 50 & 115 & 117 & 59 & 21 \\
\hline 13,81 & 31,77 & 32,32 & 16,30 & 5,80 \\
\hline
\end{tabular}

Fonte: dados da pesquisa

Na tabela 1 destaca-se a superioridade quantitativa de mulheres, com $61,26 \%$ dos respondentes. Ao analisar a faixa etária a predominância foi de jovens, sendo $31,40 \%$ para até 20 anos, e 21 a 25 anos $(37,47)$, que juntos totalizam $68,87 \%$ da amostra. A média no desempenho acadêmico foi de $6,59 \mathrm{com}$ desvio padrão de 1,71. $O$ tempo de estudo diariamente foi de até 60 minutos, para 64,05\% dos respondentes.

\section{Análise e discussões dos resultados}

$\mathrm{Na}$ tabela 2 identificou-se as estratégias metacognitivas de aprendizagem autorregulada utilizadas pelos estudantes para enfrentar suas atividades acadêmicas. Destaca-se que nas duas últimas colunas apresenta-se uma totalização das frequências, para respostas menores que quatro e maiores que quatro.

Tabela 2 -

Estratégias de aprendizagem autorregulada dos discentes.

\begin{tabular}{|c|c|c|c|}
\hline \multirow{2}{*}{\multicolumn{2}{|c|}{ Estratégia }} & \multicolumn{2}{|c|}{ Respostas } \\
\hline & & Menor que 4 & Maior que 4 \\
\hline E1 - estratégia de auto avaliação & 364 & $\begin{array}{c}66 \\
18,13 \%\end{array}$ & $\begin{array}{c}230 \\
63,19 \%\end{array}$ \\
\hline E2 - estratégia de organização e transformação & 364 & $\begin{array}{c}136 \\
37,36 \%\end{array}$ & $\begin{array}{c}181 \\
49,73 \%\end{array}$ \\
\hline $\begin{array}{l}\text { E3 - estratégia de estabelecimento de objetivos e } \\
\text { planejamento }\end{array}$ & 363 & $\begin{array}{c}159 \\
43,80 \%\end{array}$ & $\begin{array}{c}138 \\
38,02 \%\end{array}$ \\
\hline E4 - estratégia de procura de informação & 364 & $\begin{array}{c}98 \\
26,92 \%\end{array}$ & $\begin{array}{c}236 \\
64,84 \%\end{array}$ \\
\hline E5 - estratégia de tomada de apontamentos & 364 & $\begin{array}{c}73 \\
20,05 \%\end{array}$ & $\begin{array}{c}231 \\
63,46 \%\end{array}$ \\
\hline E6 - estratégia de estrutura ambiental & 364 & $\begin{array}{c}71 \\
19,51 \%\end{array}$ & $\begin{array}{c}263 \\
72,25 \%\end{array}$ \\
\hline E7 - estratégia auto consequência & 364 & $\begin{array}{c}274 \\
75,27 \%\end{array}$ & $\begin{array}{c}58 \\
15,93 \%\end{array}$ \\
\hline E8 - estratégia de repetição e memorização & 364 & $\begin{array}{c}118 \\
32,42 \%\end{array}$ & $\begin{array}{c}129 \\
35,44 \%\end{array}$ \\
\hline E9 - estratégia de procura de ajuda social & 364 & $\begin{array}{c}63 \\
17,31 \%\end{array}$ & $\begin{array}{c}263 \\
72,25 \%\end{array}$ \\
\hline E10 - estratégia de revisão de dados & 362 & $\begin{array}{c}59 \\
16,30 \%\end{array}$ & $\begin{array}{c}252 \\
69,61 \%\end{array}$ \\
\hline
\end{tabular}

Fonte: dados da pesquisa. 
Como resultado verificou-se que as estratégias metacognitivas que os estudantes utilizam preferencialmente para enfrentar suas atividades acadêmicas foram E9 (ajuda social) e E6 (estratégia de estrutura ambiental), E10 (revisão de dados), E4 (estratégia de procura de informação) e E1 (auto-avaliação). Destaca-se que a predominância do emprego das estratégias metacognitivas possui maior distribuição acima do ponto médio, à exceção da E3 (objetivos e planejamento), e principalmente de E7 (auto consequência), parcialmente convergentes aos estudos do Lima Filho, Lima \& Bruni (2016), Silva (2016) e Silva, Lay, Biavatti e Haag (2016).

Os resultados apontam que os estudantes de contabilidade não empregam o estabelecimento de objetivos e planejamento, como também a auto consequência. Ao considerar que o processo de autorregulação se concretiza de forma aberta e cíclica, o não uso dessas estratégias podem comprometer as demais fases - execução e controle e autorreflexão -, conforme destaca Silva (2016). Para Demetriou (2000) a participação ativa requer consciência dos objetivos a serem alcançados, reconhecimento das demandas da ação a se alcançar, separação e estabelecimento dos recursos internos e externos para a execução da ação, avaliação do nível de realização e alteração dos procedimentos utilizados se o resultado não for o previsto.

O passo seguinte foi analisar a relação entre as variáveis demográficas e as estratégias de aprendizagem, conforme pode ser observado no quadro 2.

Quadro 2 -

Detalhamento das variáveis demográficas e as estratégias de aprendizagem.

\begin{tabular}{|c|l|l|}
\hline Variável & \multicolumn{1}{|c|}{ Função } & \multicolumn{1}{|c|}{ Questão } \\
\hline Desempenho & Independente & Índice de desempenho acadêmico \\
\hline Bolsa & Independente & Dummy; Não = 0, Sim =1 \\
\hline Idade & Independente & \\
\hline Impostor & Independente & \\
\hline Gênero & Independente & Dummy; Feminino = 0, e Masculino =1 \\
\hline Tempo & Independente & Tempo de Estudo diário \\
\hline E1 & Dependente & $\begin{array}{l}\text { Após realização de um trabalho, sempre me certifico } \\
\text { de que parece ser bom. }\end{array}$ \\
\hline E2 & Dependente & $\begin{array}{l}\text { Procuro sempre elaborar um plano (esquema) antes } \\
\text { de iniciar um trabalho }\end{array}$ \\
\hline E3 & Dependente & $\begin{array}{l}\text { Se tenho prova, começo a estudar o mais cedo } \\
\text { possível, para ficar descansado e tranquilo no dia. }\end{array}$ \\
\hline E5 & Dependente & $\begin{array}{l}\text { Antes de iniciar um trabalho, recorro sempre à } \\
\text { biblioteca (e outros meios de pesquisa seja físico ou } \\
\text { digital) parar separar o máximo de informação sobre o } \\
\text { tema. }\end{array}$ \\
\hline E6 & Dependente & $\begin{array}{l}\text { Sempre procuro anotar o máximo de apontamento de } \\
\text { um texto lido ou da aula expositiva do professor. }\end{array}$ \\
\hline Dependente & $\begin{array}{l}\text { Para ter melhor concentração, procuro sempre } \\
\text { ambiente que não proporcione distração. }\end{array}$ \\
\hline Dependente & $\begin{array}{l}\text { Quando faço uma prova, se ocorrer bem, ofereço-me } \\
\text { recompensa; caso ocorra o contrário, abro mão de } \\
\text { algo que tanto queria. }\end{array}$ \\
\hline & De \\
\hline
\end{tabular}




\begin{tabular}{|c|l|l|}
\hline E8 & Dependente & $\begin{array}{l}\text { Uso estratégias para memorizar pontos (ou fórmulas) } \\
\text { ou tentar decorar o assunto a ser estudado. }\end{array}$ \\
\hline E9 & Dependente & $\begin{array}{l}\text { Quando os problemas surgem e eu não posso } \\
\text { resolvê-los sozinho, eu procuro ajuda externa. }\end{array}$ \\
\hline E10 & Dependente & $\begin{array}{l}\text { Avalio o meu desempenho, vejo que devo melhorar a } \\
\text { fim de me preparar para uma prova. }\end{array}$ \\
\hline EMed & Dependente & Média de Uso das Estratégias E1 a E10 \\
\hline
\end{tabular}

Numa regressão múltipla inicial, com todas as variáveis, predominaram variáveis não significativas. Mas a análise individualizada em stepwise dos fatores dependentes permite uma maior aproximação entre todos, por conta da identificação de características muito comuns. Isso pode ser melhor visualizado na tabela 4.

Tabela 3 -

Resultados das regressões múltiplas.

\begin{tabular}{c|c|c|c|c|c|c|c}
\hline Dependente & Bolsa & Desemp & Idade & Impostor & Genero & Tempo & $\begin{array}{c}\text { Adj R2 } \\
\text { Prob }>\mathrm{F}\end{array}$ \\
\hline E.Média & & & & & .2792307 & .34258 & 0.1299 \\
& & & & & 0.011 & 0.000 & 0.0000 \\
\hline E1 & -.241252 & & .2848157 & & & .4152867 & 0.1449 \\
& 0.040 & & 0.000 & & & 0.000 & 0.0000 \\
\hline E2 & & & & & & .3036779 & 0.0284 \\
& & & & & & 0.001 & 0.0008 \\
\hline E3 & & .1334259 & & & & .5329605 & 0.1129 \\
& & 0.014 & & & & 0.000 & 0.0000 \\
\hline E4 & & & & & & .4606717 & 0.0593 \\
& & & & & & 0.000 & 0.0000 \\
\hline E5 & & & & & .4592411 & .4006334 & 0.0857 \\
& & & & & .6909531 & 0.000 & 0.0000 \\
\hline E6 & -.427546 & & & & 0.000 & & 0.0537 \\
& 0.004 & & & .0137937 & & .2576941 & 0.0000 \\
\hline E7 & & & & 0.000 & & 0.002 & 0.0000 \\
\hline E8 & & .1252356 & & .0086479 & & .5950187 & 0.1277 \\
& & 0.026 & & 0.034 & & 0.000 & 0.0000 \\
\hline E9 & & & & - & & & 0.0471 \\
& & & & .7064262 & & & 0.0001 \\
& & & & 0.000 & & & \\
\hline E10 & & & & & .589742 & .17853 & 0.0403 \\
& & & & & 0.001 & 0.022 & 0.0003 \\
\hline
\end{tabular}

Fonte: dados da pesquisa

Considerando a média de uso das estratégias de aprendizagem, apareceram significativas para explicar a intensidade de uso de estratégias o gênero e o tempo, em que o gênero masculino demonstrou maior emprego médio de estratégias de aprendizagem. 
Como pode ser observado o tempo de estudo está associado ao emprego de oito, das dez estratégias de ensino autorregulado estudadas, o que pode demonstrar a importância dos alunos em terem tempo suficiente de estudarem, planejarem seus estudos e avaliarem adequadamente os resultados obtidos.

Como outras pesquisas já demonstraram (Carson, 2011; Puzziferro, 2008; Silva et al., 2019), existe uma correlação positiva entre a capacidade do aluno de autorregular seus estudos com o seu desempenho acadêmico. Por esse motivo, as discussões sobre currículos mais flexíveis e que permitam que os estudos tenham um maior tempo de estudo fora de sala de aula parecem fundamentadas.

A Estratégia 1, de auto avaliação, está positivamente associada com a idade, o que indica que quanto mais velho é o estudante, mais ele se preocupa em certificar-se que um trabalho realizado está bom. Os resultados demonstram que essa é a única estratégia significativamente associada com a idade, o que demonstra que os usos de todas as outras estratégias independem da idade dos respondentes. Esse achado pode ser particularmente útil para os docentes, já que eles podem direcionar esforços com o objetivo de mostrar aos alunos mais novos a importância de revisarem adequadamente seus trabalhos antes do seu envio final, talvez até mesmo citando como exemplo os alunos mais velhos que possuem o costume de tal prática. Além disso, esses achados corroboram as pesquisas de Carson (2011), Chang (2007) e Puzziferro (2008).

A Estratégia 2, relacionada ao planejamento dos estudos, e a Estratégia 4, de coletar outras fontes de informação, possuem relação significativa somente com o tempo. Essas estratégias estão relacionadas ao processo fundamental da administração, que é o planejamento, e que pode refletir nas demais estratégias e por consequência no resultado.

Esses resultados reforçam que não apenas a disponibilidade de tempo, mas o planejamento de como empregar o tempo para o desenvolvimento das atividades pode contribuir com o desempenho. Nos estudos de Carson (2011), Puzziferro (2008) e Silva et al. (2019) foi relacionado que o tempo é fator significante para o desempenho, enquanto aqui, ao comparar as estratégias com as características pode-se identificar que a disponibilidade está relacionada ao planejamento e organização do tempo.

A Estratégia 3, de buscar realizar estudos com maior antecedência, possui relação significativa com o desempenho, o que parece demonstrar a relação direta do desempenho prévio relacionando a manutenção de um status de desempenho superior. Este resultado pode contribuir para justificar os estudos sobre a procrastinação acadêmica.

Ao fim, alunos de menor desempenho relacionam-se a uma menor antecipação dos estudos, que por sua vez tem como consequência um menor desempenho, gerando um ciclo vicioso. Assim como já indicado essa estratégia possui forte correlação com a disponibilidade de tempo, indicada anteriormente (Carson, 2011; Puzziferro, 2008; Silva et al., 2019).

A adoção da Estratégia 5, do emprego de anotações, relaciona-se, além do tempo, ao gênero, sendo que o masculino estaria associado a essa estratégia, assim como a Estratégia 6, de busca por ambientes sem distrações. Esses achados corroboram a relação positiva de Puzziferro (2008) na forma fraca, o que poderia ser possivelmente explicada por limitar-se a um gênero. Isto também pode denotar o reconhecimento de 
uma dificuldade de concentração, o que exigiria a adoção das referidas estratégias, ou que os indivíduos do gênero feminino, ao optar por outras estratégias, não valorizariam estas.

A Estratégia 10, da auto-avaliação, que se relaciona positivamente ao gênero masculino. Esse achado pode denotar uma quebra de paradigma de que o gênero masculino pouco planeja seus estudos. Esses resultados também podem explicar a divergência entre os resultados de Carson (2011), Cho e Shen (2013) e Puzziferro (2008), em relação a ChanLin (2012), que não confirmaram a relação dessa estratégia com o desempenho na sua amostragem.

A Estratégia 7, de auto recompensa, a 8 da Memorização, e a 9 da busca por auxílio estão relacionadas significativamente à auto percepção de impostor. Estes resultados podem ter apego ao imaginário de que a memorização não seria um aprendizado significativo, mas temporário, da mesma forma a auto recompensa é uma conduta de busca por reconhecimento, evitando a busca por auxílio de terceiros, já que tais traços se relacionam a um indivíduo centralizador e auto suficiente.

Embora a memorização esteja relacionada a percepção de impostor, também se relaciona de forma mediata ao desempenho prévio, ou seja, indivíduos de melhor desempenho buscam a adoção dessa estratégia para manter seus resultados ou por reconhecer que um conhecimento mesmo que temporário pode ensejar benefícios no longo prazo.

Esses resultados precisam ser pesquisados por outros vieses, visto que a relação da memorização com o desempenho posterior tem apresentado divergência na literatura (Puzziferro, 2008; Klingsieck et al., 2012; Wang \& Wu, 2008). Interessante destacar que o pensamento crítico ou capacidade de examinar cuidadosamente o material de aprendizagem (Richardson et al., 2012) também foi identificado com influência positiva e fraca (Puzziferro, 2008) em relação do desempenho acadêmico. No que tange a relação entre a busca de ajuda, relaciona-se a uma maior percepção de não-impostor, ou maior autenticidade, que também relaciona-se ao desempenho posterior (ChanLin, 2012; Puzziferro, 2008).

\section{Conclusões}

Analisar a adoção de estratégias metacognitivas de aprendizagem autorregulada relacionando-as as características dos indivíduos é relevante, pois algumas estratégias são manifestas como determinantes de um melhor desempenho (Silva et al., 2019) indicam que o desempenho está associado apenas a algumas estratégias de aprendizagem, mas não é conhecido na literatura a validação empírica das variáveis que levam a adoção de determinada estratégia.

A aplicação do instrumento elaborado por Zimmermann e Ponz (1986), de dez afirmações relativas ao uso das estratégias metacognitivas, revelou as estratégias mais adotadas entre os estudantes de Ciências Contábeis: evitar ambientes sem distração, busca de auxílio para resolução de problemas e auto avaliação do desempenho para preparação para uma prova. As estratégias reveladas como de menor adoção foram o estudo com maior antecedência, da auto-recompensa e da memorização, destas, duas relacionadas significativamente com ao desempenho. 
Os resultados estatísticos também revelam a relação entre as variáveis das características individuais às estratégias, sendo que a característica determinante foi a disponibilidade de tempo positiva e significante com oito das dez estratégias. As relações atribuídas à adoção de bolsas para incentivo ao desempenho parecem conflitantes com o comportamento esperado, enquanto o gênero masculino foi associado com estratégias que evidenciam uma quebra de paradigmas, ou ao menos um desuso da amostra feminina de algumas estratégias.

O desempenho e caracterização de impostor relacionou-se a uma quantidade relevante de estratégias entre si, como a adoção da memorização para ambos, e auto recompensa ao auto-suficiência do impostor. Esses achados podem contribuir para as discussões sobre a síndrome do impostor entre os estudantes de Ciências Contábeis. A idade somente foi significativa à adoção da estratégia de revisão das tarefas.

A pesquisa contribui para revelar relações estatísticas sobre a discussão de fatores típicos da discussão do ponto de vista da administração pedagógica. Esses achados podem contribuir com implicações práticas na gestão pedagógica de cursos de graduação e no planejamento de aulas, ao relacionar os perfis da turma às possibilidades de atividades, bem como a política de inclusão e retenção de alunos. Do ponto de vista teórico, os resultados identificados podem explicar relações divergentes ou de significância fraca da explicação do desempenho discente na literatura referenciada.

Estas evidências abrem lacunas para novas pesquisas e para a verificação em outras amostras, principalmente relacionando as estratégias ao desempenho do discente (Silva et al., 2019), bem como sobre os fatores qualitativos que relacionam as características individuais abordadas superficialmente neste trabalho com a adoção das estratégias. Os resultados, se verificados em outras amostras, ambientes e culturas, podem suprir uma lacuna relevante na literatura de aprendizagem autoregulada.

\section{Referências}

AMERICAN ACCOUNTING ASSOCIATION. The pathways commission charting a national strategy for the next generation of accountants. Lakewood Ranch, FL: AAA, 2012.

AICPA - American Institute of Certified Public Accountants. The AICPA core competency frame-work for entry into the accounting profession, Carolina do Norte: AICPA, 2000.

BECKER, Lana. Self-regulated learning interventions in the introductory accounting course: An empirical study. Issues in Accounting Education, Lakewood Ranch, FL, v. 28, n. 3, 2013, p. 435-460.

BOLZAN, Giovana et al. Accounting teaching and the convergence process to international financial reporting standards. Revista Ambiente Contábil, Natal, v. 12, n. 1, 2020, p. 130-151.

BROADBENT, Jaclyn; POON, Walter. Self-regulated learning strategies \& academic achievement in online higher education learning environments: A systematic review. The Internet and Higher Education, Londres: Elsevier, v. 27, 2015, p. 1-13.

BYRNE, Marann; FLOOD, Barbara; WILLIS, Pauline. An Inter-Institutional Exploration of the Learning Approaches of Students Studying Accounting. International Journal of Teaching and Learning in Higher Education, Londres: Elsevier, v. 20, n. 2, 2009, p. 155167. 
CARSON, Andrew. Predicting student success from the Lassi for learning online (LLO). Journal of Educational Computing Research, Thousand Oaks: Sage, v. 45, n. 4, p. 399414, 2011.

CHANG, Mei-Mei. Enhancing web-based language learning through self-monitoring. Journal of Computer Assisted Learning, Place Malden: Blackwell Publishing, v. 23, n. 3, 2007, p. 187-196.

CHANLIN, Lih-Juan. Learning strategies in web-supported collaborative project. Innovations in Education and Teaching International, Londres: Routledge, v. 49, n. 3, 2012, p. 319-331.

$\mathrm{CHO}$, Moon-Heum; SHEN, Demei. Self-regulation in online learning. Distance education, Londres: Taylor \& Francis, v. 34, n. 3, 2013, p. 290-301.

COLEMAN, James. The concept of equality of educational opportunity. Harvard educational review, Massachusetts: Harvard, v. 38, n. 1, 1968, p. 7-22.

DEMETRIOU, Andreas. Organization and development of self-understanding and selfregulation: Toward a general theory. In: Handbook of self-regulation. Massachusetts: Academic Press, 2000, p. 209-251.

EFFENEY, Gerard; CARROLL, Annemaree; BAHR, Nan. Self-Regulated Learning: Key strategies and their sources in a sample of adolescent males. Australian Journal of Educational \& Developmental Psychology, NewCastle: University of Newcastle, v. 13, 2013, p. 58-74.

FARIAS, Rafael et al. Dificuldades dos professores do curso de Ciências Contábeis. Revista Docência do Ensino Superior, Belo Horizonte, v. 9, 2019, p. 1-20.

FLAVELL, John. Metacognitive aspects of problem solving. In: RESNICK, Lauren B (ed.). The nature of intelligence. Hillsdale: Lawrence Erlbaum, 1976, p. 231-235.

FORNELL, Claes; LARCKER, David F. Evaluating structural equation models with unobservable variables and measurement error. Journal of marketing research, Chicago: AMA, v. 18, n. 1, 1981, p. 39-50.

GIMÉNEZ, Víctor; PRIOR, Diego; THIEME, Claudio. Technical efficiency, managerial efficiency and objective-setting in the educational system: an international comparison. Journal of the Operational Research Society, Londres: Taylor \& Francis, v. 58, n. 8, 2007, p. 996-1007.

HAIR, Josep et al. Análise multivariada de dados. Porto Alegre: Bookman, 2009.

HENSELER, Jörg; RINGLE, Christian M; SINKOVICS, Rudolf R. The use of partial least squares path modeling in international marketing. In: New challenges to international marketing. Bingley: Emerald, 2009.

HODGES, Charles; KIM, Chanmin. Email, self-regulation, self-efficacy, and achievement in a college online mathematics course. Journal of Educational Computing Research, Thousand Oaks: Sage, v. 43, n. 2, 2010, p. 207-223.

KLINGSIECK, Katrin et al. Procrastination in a distance university setting. Distance Education, Londres: Taylor \& Francis, v. 33, n. 3, 2012, p. 295-310.

KRAUSS, Jessica; SILVA, Thiago; ZONATTO, Vicente. EVIDÊNCIAS DOS EFEITOS DAS ESTRATÉGIAS METACOGNITIVAS DE APRENDIZAGEM AUTORREGULADA NA FORMAÇÃO DE INCONSCIENTE COLETIVO NO CONHECIMENTO DE CONTABILIDADE DE CUSTOS, 11, 2017. Anais ... Belo Horizonte: Anpcont, 2017, p. 920. 
LIMA FILHO, Raimundo Nonato; BRUNI, Adriano Leal. Metacognição estimula características empreendedoras? Uma análise em profissionais de administração. Race: Revista de Administração, Contabilidade e Economia, Joaçaba, v. 14, n. 2, 2015, p. 427450.

NATIONAL RESEARCH COUNCIL. Grand challenges in environmental sciences. Washington: National Academies Press, 2001.

OLIVEIRA, Katya; BORUCHOVITCH, Evely; SANTOS, Acácia Aparecida. Estratégias de aprendizagem e desempenho acadêmico: evidências de validade. Psicologia: teoria $e$ pesquisa, Brasília: Scielo, v. 25, n. 4, 2009, p. 531-536.

PUZZIFERRO, Maria. Online technologies self-efficacy and self-regulated learning as predictors of final grade and satisfaction in college-level online courses. The Amer. Jrnl. of Distance Education, Nova Iorque, v. 22, n. 2, 2008, p. 72-89.

RAVENSCROFT, Susan; WAYMIRE, Tammy; WEST, Timothy. Accounting students' metacognition: The association of performance, calibration error, and mindset. Issues in Accounting Education, Londres: Elsevier, v. 27, n. 3, 2012, p. 707-732.

RICHARDSON, Michelle; ABRAHAM, Charles; BOND, Rod. Psychological correlates of university students' academic performance: a systematic review and meta-analysis. Psychological bulletin, Washington: APA, v. 138, n. 2, 2012, p. 353.

RINGLE, Christian; WENDE, Sven; BECKER, Jan-Michael. SmartPLS 3. Bönningstedt: SmartPLS, 2015.

ROSÁRIO, Pedro. Diferenças processuais na aprendizagem: avaliação alternativa das estratégias de auto-regulação da aprendizagem. Psicologia, Educação e Cultura, Gaya: ISPGAya, v. 5, n. 1, 2001, p. 87-102.

ROSÁRIO, Pedro. Estudar o estudar: as (des)venturas do testas. Porto: Porto, 2004.

SALLABERRY, Jonatas; SALLABERRY, Bárbara. A acessibilidade sob o enfoque ético na contabilidade. Revista Ambiente Contábil, Natal, v. 4, n. 1, 2012, p. 89-105.

SCHLEIFER, Lydia; DULL, Richard. Metacognition and performance in the accounting classroom. Issues in Accounting Education, Londres: Taylor \& Francis, v. 24, n. 3, 2009, p. 339-367.

SILVA, Thiago. Estratégia metacognitiva de aprendizagem autorregulada, percepção docente sobre a aprendizagem e métodos educacionais em contabilidade: estudo de caso em uma universidade pública catarinense. Blumenau: Furb, 2016. 97f. Dissertação de Mestrado (Mestrado em Ciências Contábeis). Programa de Pós-Graduação em Ciências Contábeis da Universidade Regional de Blumenau.

SILVA, Thiago, et al. Academic performance and the level of use of metacognitive selfregulatory learning strategies by accounting students. American Accounting Association Meeting, San Francisco: AAA, 2019.

SILVA, Thiago, et al. Estratégias de aprendizagem autorregulada em contabilidade: um estudo em duas instituições privadas do ensino superior catarinense. Revista Mineira de Contabilidade, Belo Horizonte, v. 17, n. 3, 2016, p. 5-15.

SMITH, Pamela. Understanding self-regulated learning and its implications for accounting educators and researchers. Issues in Accounting Education, Lakewood: AAA, v. 16, n. 4, 2001, p. 663-700. 
SPERLING, Rayne; HOWARD, Bruce; STALEY, Richard; DUBOIS, Nelson. Metacognition and self-regulated learning constructs. Educational Research and Evaluation, Londres: Taylor \& Francis, v. 10, n. 2, 2004, p. 117-139.

TUYSUZOGLU, Banu. An investigation of the role of metacognitive behavior in selfregulated-learning when learning a complex science topic with a hypermedia. Chapel Hill: University of North Carolina, 2011. (Doctoral dissertation). The University of North Carolina.

WANG, Shu-Ling; WU, Pei-Yi. The role of feedback and self-efficacy on web-based learning: The social cognitive perspective. Computers \& Education, Londres: Elsevier, v. 51, n. 4, 2008, p. 1589-1598.

WANG, Ying et al. Characteristics of distance learners: Research on relationships of learning motivation, learning strategy, self-efficacy, attribution and learning results. Open Learning: The Journal of Open, Distance and e-Learning, Londres: Taylor \& Francis, v. 23, n. 1, 2008, p. 17-28.

ZIMMERMAN, Barry. Attaining self-regulation: A social cognitive perspective. In: Handbook of self-regulation. Massachusetts: Academic Press, 2000, p. 13-39.

ZIMMERMAN, Barry. Self-regulated learning and academic achievement: An overview. Educational psychologist, Londres: Taylor \& Francis, v. 25, n. 1, 1990, p. 3-17.

ZIMMERMAN, Barry. Theories of self-regulated learning and academic achievement: An overview and analysis. In: ZIMMERMAN, Barry; SCHUNK, Dale H (eds.). Self-regulated learning and academic achievement. Londres: Routledge, 2013, p. 10-45.

ZIMMERMAN, Barry; PONS, Manuel Martinez. Development of a structured interview for assessing student use of self-regulated learning strategies. American Educational Research Journal, Thousand Oaks: Sage, v. 23, n. 4, 1986, p. 614-628.

Thiago Bruno de Jesus Silva é professor na Universidade Federal da Grande Dourados.

Orcid: http://orcid.org/0000-0002-1128-6601.

Endereço: Campus Reitor João David Ferreira Lima, s/n - 88040-900 - Florianópolis - SC - Brasil.

E-mail: thiagobsilva@ufgd.edu.br.

Marcelo Machado de Freitas é estudante no curso de doutorado em Contabilidade na Universidade Federal de Santa Catarina.

Orcid: http://orcid.org/0000-0001-9767-7285.

Endereço: Campus Reitor João David Ferreira Lima, s/n - 88040-900 - Florianópolis - SC - Brasil.

E-mail: mmf.marcelofreitas@gmail.com.

Jonatas Dutra Sallaberry é estudante no curso de doutorado em Contabilidade pela Universidade Federal de Santa Catarina.

Orcid: http://orcid.org/0000-0001-7492-727X.

Endereço: Campus Reitor João David Ferreira Lima, s/n - 88040-900 - Florianópolis - SC - Brasil.

E-mail: jonatas.sallaberry@hotmail.com. 
Leonardo Flach é professor na Universidade Federal de Santa Catarina.

Orcid: http://orcid.org/0000-0002-4316-0704.

Endereço: Campus Reitor João David Ferreira Lima, s/n - 88040-900 - Florianópolis - SC - Brasil.

E-mail: leonardo.flach@gmail.com.

Recebido em 4 de fevereiro de 2020.

Aceito em 19 de abril de 2020.

(c) (i) 